\title{
UN THÉORÈME DE L'APPLICATION OUVERTE SUR LES CORPS VALUÉS ALGÉBRIQUEMENT CLOS
}

\author{
LAURENT MORET-BAILLY*
}

\section{Résumé}

Soit $K$ un corps algébriquement clos muni d'une valeur absolue non triviale, et soit $f: X \rightarrow Y$ un morphisme de $K$-schémas de type fini. On montre que $f$ est universellement ouvert si et seulement si l'application induite sur les points $K$-rationnels est ouverte pour les topologies déduites de la valeur absolue.

\begin{abstract}
Let $K$ be an algebraically closed field with a nontrivial absolute value, and let $f: X \rightarrow Y$ be a morphism of $K$-schemes of finite type. We show that $f$ is universally open if and only if the induced map on $K$-rational points is open for the topologies deduced from the absolute value.
\end{abstract}

\section{Introduction}

Soit $(K,||$.$) un corps valué (c'est-à-dire que ||:. K \rightarrow \mathrm{R}$ est une valeur absolue sur $K$, qui sera supposée non triviale). Pour tout $K$-schéma de type fini $X$, on notera $X_{\text {top }}$ l'ensemble $X(K)$ muni de la topologie «forte» déduite de la valeur absolue.

THÉORÈme 1.1. Soit $(K,||$.$) un corps valué algébriquement clos, et soit$ $f: X \rightarrow Y$ un morphisme de $K$-schémas de type fini. Les conditions suivantes sont équivalentes:

(i) $f$ est universellement ouvert;

(ii) l'application continue $f_{\text {top }}: X_{\text {top }} \rightarrow Y_{\text {top }}$ induite par $f$ est ouverte.

Remarques (1) Soit $f: X \rightarrow Y$ un morphisme localement de type fini de schémas localement noethériens. Rappelons que $f$ est universellement ouvert dans les cas suivants:

(i) $f$ est plat (ou, plus généralement, il existe un $\mathscr{O}_{X}$-module cohérent et $f$-plat, de support $X)$, cf. [7], (2.4.6);

\footnotetext{
* L'auteur est membre du projet «Points entiers et points rationnels» de l'Agence nationale pour la recherche.

Received 23 March 2011, in final form 30 May 2011.
} 
(ii) $Y$ est géométriquement unibranche et $f$ est équidimensionnel («critère de Chevalley» [8], (14.4.4)). On peut d'ailleurs, pour beaucoup de questions, se ramener à ce cas en utilisant par exemple ([8], (14.4.9)).

(2) Tout morphisme plat $f: X \rightarrow Y$ d'espaces analytiques complexes est ouvert; ce résultat semble dû à Douady ([6], corollaire à la fin de l'article). On en trouvera une démonstration plus élémentaire dans [1] (V, Theorem 2.12), ou [2] (V, théorème 2.10), qui montre en outre (sans hypothèse de platitude) que si $Y$ est localement irréductible et $f$ équidimensionnel, alors $f$ est ouvert: on reconnaît l'analogue de la condition (ii) de (1).

(3) Tout morphisme plat d'espaces analytiques rigides (quasi-compacts et quasi-séparés) est ouvert ([3], cor. 7.2); la démonstration utilise le cas d'un morphisme de schémas via les modèles formels.

(4) Selon V. Berkovich (non publié, communication privée), si $f: X \rightarrow Y$ est un morphisme sans bord d'espaces analytiques non archimédiens, et si $\mathscr{F}$ est un $\mathscr{O}_{X}$-module cohérent et $f$-plat, alors la restriction de $f$ au support de $\mathscr{F}$ est une application ouverte.

(5) $\mathrm{Vu}$ les trois remarques précédentes, la partie «(i) $\Rightarrow$ (ii)» du théorème 1.1 est essentiellement connue dans le cas plat (et dans le cas complexe); les seuls mérites de la démonstration présentée ici sont (outre le cas ultramétrique non plat) de ne pas traiter séparément les cas archimédien et non archimédien, et de ne pas recourir aux espaces analytiques sous-jacents. Quant à la partie «(ii) $\Rightarrow$ (i)» c'est un simple exercice, que je n'ai toutefois pas trouvé dans la littérature.

(6) Je remercie Vladimir Berkovich, Siegfried Bosch, Antoine Ducros, Francesco Polizzi et le rapporteur pour leurs remarques.

\section{Rappels et préliminaires}

\subsection{Corps topologiques}

Soit $K$ un corps topologique; on supposera toujours dans la suite que la topologie de $K$ n'est pas grossière, ce qui implique que $K$ est séparé (démonstration: $K$ admet un ouvert non vide et strict; par translation, il admet un ouvert non vide $U \subset K^{*}$; donc $K^{*}=\bigcup_{\lambda \in K^{*}} \lambda U$ est ouvert, donc $\{0\}$ est fermé).

Pour tout $K$-schéma de type fini $X$, on sait alors munir l'ensemble $X(K)$ d'une topologie dite forte de telle sorte que, si l'on note $X_{\text {top }}$ l'espace topologique obtenu:

- si $X=\mathrm{A}_{K}^{1}$, la topologie forte sur $X_{\text {top }}=K$ est celle de $K$;

- tout $K$-morphisme $f: X \rightarrow Y$ de $K$-schémas de type fini induit une application continue $f_{\text {top }}: X_{\text {top }} \rightarrow Y_{\text {top }}$, qui est de plus un plongement topologique ouvert (resp. fermé) si $f$ est une immersion ouverte (resp. fermée); 
- si $X$ et $Y$ sont deux $K$-schémas de type fini, la bijection canonique $\left(X \times{ }_{K} Y\right)_{\text {top }} \rightarrow X_{\text {top }} \times Y_{\text {top }}$ est un homéomorphisme.

Ces conditions entraînent encore que toute immersion induit un plongement topologique, et que la formation de $X_{\text {top }}$ commute aux produits fibrés.

Nous aurons à considérer les propriétés suivantes d'un corps topologique $K$ :

(H) Pour tout $K$-morphisme étale $f: X \rightarrow Y$ de $K$-schémas de type fini, l'application induite $f_{\text {top }}: X_{\text {top }} \rightarrow Y_{\text {top }}$ est ouverte.

(F) Pour tout $K$-morphisme fini $f: X \rightarrow Y$ de $K$-schémas de type fini, l'application induite $f_{\text {top }}: X_{\text {top }} \rightarrow Y_{\text {top }}$ est fermée.

Proposition 2.1.1. Soit $K$ un corps topologique non discret. Soit $X$ un $K$-schéma de type fini irréductible, et soit $\Omega$ un ouvert non vide de $X_{\text {top. }}$. Alors, dans chacun des cas suivants, $\Omega$ est dense dans $X$ pour la topologie de Zariski:

(i) $X=\mathrm{A}_{K}^{n}$;

(ii) $K$ vérifie $(\mathrm{H})$ et $X$ est lisse sur $K$;

(iii) $K$ vérifie $(\mathrm{H})$ et est algébriquement clos.

DÉmonstration. Dans le cas (i), l'hypothèse que $K$ est non discret entraîne directement le résultat si $n=1$ (tout ouvert non vide de $K$ est infini). Le cas $n \geq 1$ s'en déduit grâce au fait bien connu suivant: si $I_{1}, \ldots, I_{n}$ sont des parties infinies de $K$, tout polynôme $F \in K\left[T_{1}, \ldots, T_{n}\right]$ nul sur $\prod_{k=1}^{n} I_{k}$ est nul.

Dans le cas (ii), soit $x$ un point de $\Omega$. Il existe un voisinage $V$ de $x$ dans $X$ et un morphisme étale $\pi: V \rightarrow \mathrm{A}_{K}^{n}$. D'après $(\mathrm{H}), \pi(\Omega)$ est un ouvert non vide de $K^{n}$ donc est Zariski-dense dans $\mathrm{A}_{K}^{n}$, ce qui entraîne immédiatement la conclusion.

Dans le cas (iii), soit $x$ un point de $\Omega$. Il suffit de montrer que pour toute courbe irréductible $C \subset X$ contenant $x$, l'ensemble $\Omega \cap C$ est infini. Nous sommes donc ramenés au cas où $X$ est une courbe, et même au cas déjà traité d'une courbe lisse en passant à la normalisée (puisque $K$ est algébriquement clos, la normalisation induit une surjection sur les points rationnels).

\subsection{Corps valués}

Tout corps valué $(K,||$.$) sera considéré comme corps topologique, la topo-$ logie étant celle de la distance $(x, y) \mapsto|x-y|$ sur $K$. On sait qu'alors le complété $\widehat{K}$ de $K$ est encore de façon naturelle un corps valué, algébriquement clos si $K$ l'est. 
Proposition 2.2.1. Soit $(K,||$.$) un corps valué.$

(i) Si K est séparablement fermé dans $\widehat{K}$, il vérifie la condition $(\mathrm{H})$ de 2.1 . Plus précisément, tout $K$-morphisme étale $f: X \rightarrow Y$ de $K$-schémas de type fini induit un homéomorphisme local $f_{\text {top }}: X_{\text {top }} \rightarrow Y_{\text {top. }}$.

(ii) Si $K$ est algébriquement fermé dans $\widehat{K}$, il vérifie la condition $(\mathrm{F})$ de 2.1 . En particulier, tout corps valué complet ou algébriquement clos vérifie $(\mathrm{H})$ et (F).

Rappelons brièvement la démonstration: on se ramène, par des dévissages convenables, au cas où $Y=\operatorname{Spec} A$ est l'espace affine de dimension $n$ sur $K$ et où

$$
X= \begin{cases}\operatorname{Spec} A\left[T, \frac{1}{F^{\prime}(T)}\right] /(F(T)), F \in A[T] & \text { (cas (i)) } \\ \operatorname{Spec} A[T] /(F(T)), F \in A[T] \text { unitaire } & \text { (cas (ii)) }\end{cases}
$$

Lorsque $K$ est complet, le cas (i) est le théorème d'inversion locale classique, et le cas (ii) est la «continuité des racines» pour laquelle on renvoie par exemple à [4], § 3.4 .

Dans le cas général, notons $\widehat{f}_{\text {top }}: X(\widehat{K}) \rightarrow Y(\widehat{K})$ l'application induite par $f$ sur les points à valeurs dans $\widehat{K}$ : on remarque alors que si $K$ est séparablement (resp. algébriquement) fermé dans $\widehat{K}$, on a $\widehat{f}_{\text {top }}^{-1}(Y(K))=X(K)$ dans le cas (i) (resp. (ii)), de sorte que l'on est ramené au cas complet.

Remarque 2.2.2. La condition que $K$ soit séparablement fermé dans $\widehat{K}$ équivaut au fait que la valeur absolue $\mid$. | soit hensélienne, c'est-à-dire admette un unique prolongement à toute extension algébrique de $K$. Dans le cas non archimédien, il revient au même de dire que l'anneau de la valuation associée (i.e. la boule unité fermée de | .|) est hensélien. Voir par exemple [5], chapitre 6, exercices du $\S 8$.

Remarque 2.2.3. Supposons la valeur absolue de $K$ associée à une valuation discrète d'anneau $A$ hensélien.

Si de plus $A$ est excellent. alors $K$ est même algébriquement fermé dans $\widehat{K}$, puisque ce dernier est une extension séparable de $K$. Les conditions (H) et (F) sont donc vérifiées dans ce cas.

En revanche, il y a des exemples, en caractéristique $p>0$, où $\widehat{K}$ contient un élément $\alpha \notin K$ tel que $\alpha^{p} \in K$; dans ce cas, le morphisme de Frobenius (relatif) $f: \mathrm{A}_{K}^{1} \rightarrow \mathrm{A}_{K}^{1}$ est fini, mais $f_{\text {top }}$ n'est pas fermée puisque $\alpha^{p}$ est adhérent à $K^{p}$ sans appartenir à $K^{p}$. 


\subsection{Applications et morphismes universellement ouverts}

Commençons par un énoncé topologique élémentaire. Nous dirons qu'une application continue $p: U \rightarrow V$ est un quotient topologique si $V$ s'identifie à l'espace quotient de $U$ par la relation d'équivalence associée à $p$. C'est notamment le cas si $p$ est surjective et ouverte, ou surjective et fermée.

Proposition 2.3.1. Considérons un diagramme cartésien d'applications continues

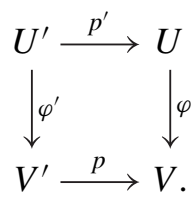

(i) Si $\varphi$ est ouverte, alors $\varphi^{\prime}$ est ouverte. (Autrement dit, les applications continues ouvertes sont universellement ouvertes).

(ii) Si p est un quotient topologique et si $\varphi^{\prime}$ est ouverte, alors $\varphi$ est ouverte.

DÉmonstration. Pour (i), on se ramène à deux cas:

- $T$ est un espace topologique, $V^{\prime}=V \times T$, et $p$ est la projection naturelle: alors $U^{\prime}=U \times T$ et l'assertion résulte de la définition de la topologie produit.

- $V^{\prime}$ est un sous-espace de $V$ et $p$ est l'inclusion: alors $U^{\prime}=\varphi^{-1}\left(V^{\prime}\right)$. Un ouvert $W^{\prime}$ de $U^{\prime}$ est de la forme $W \cap U^{\prime}$, où $W$ est un ouvert de $U$. L'assertion résulte de la «formule de projection» $\varphi\left(W \cap \varphi^{-1}\left(V^{\prime}\right)\right)=$ $\varphi(W) \cap V^{\prime}$.

Montrons (ii): si $W$ est un ouvert de $U$, alors, le diagramme étant cartésien, on a $p^{-1}(\varphi(W))=\varphi^{\prime}\left(p^{\prime-1}(W)\right)$. On en déduit que $p^{-1}(\varphi(W))$ est ouvert dans $V^{\prime}$, d'où la conclusion vu l'hypothèse sur $p$.

En ce qui concerne les morphismes universellement ouverts de schémas, nous aurons besoin de la proposition suivante, extraite de [8], (14.5.9), qui affirme l'existence de «quasi-sections» pour de tels morphismes:

Proposition 2.3.2. Soient $Y$ un schéma localement noethérien irréductible, $f: X \rightarrow Y$ un morphisme localement de type fini, y un point géométriquement unibranche (par exemple normal) de Y. Les conditions suivantes sont équivalentes:

(i) $f$ est universellement ouvert en tout point de $f^{-1}(y)$;

(ii) pour tout point fermé $x$ de $f^{-1}(y)$, il existe une partie localement fermée irréductible $X^{\prime}$ de $X$, contenant $x$, et telle que la restriction $X^{\prime} \rightarrow Y$ de $f$ soit un morphisme quasi-fini et dominant. 
Enfin, rappelons une technique de localisation étale qui permet de «rendre finis les morphismes quasi-finis»:

THÉORÈme 2.3.3 ([9], (18.12.1)). Soient $f: X \rightarrow Y$ un morphisme localement de type fini, y un point de $Y, x$ un point isolé de la fibre $f^{-1}(y)$. Alors il existe un morphisme étale $Y^{\prime} \rightarrow Y$, un point $x^{\prime}$ de $X^{\prime}=X \times_{Y} Y^{\prime}$ au-dessus de $x$, et un voisinage ouvert $V^{\prime}$ de $x^{\prime}$ dans $X^{\prime}$ tel que le morphisme composé $V^{\prime} \hookrightarrow X^{\prime} \stackrel{\mathrm{pr}_{2}}{\longrightarrow} Y^{\prime}$ soit fini.

D'après 2.2.1, le théorème 1.1 résulte des suivants, un peu plus précis:

THÉORÈme 2.4. Soit $K$ un corps topologique algébriquement clos, non discret et vérifiant $(\mathrm{H})$, et soit $f: X \rightarrow Y$ un morphisme de $K$-schémas de type fini. Si $f_{\text {top }}$ est ouverte, alors $f$ est universellement ouvert.

THÉORÈME 2.5. Soit $K$ un corps topologique algébriquement clos et vérifiant (H) et (F), et soit $f: X \rightarrow Y$ un morphisme de $K$-schémas de type fini. Si $f$ est universellement ouvert, alors $f_{\text {top }}$ est ouverte.

DÉMONSTRATION DE 2.4 (le théorème 2.5 sera démontré au paragraphe 3 ). D'après 2.3.1(i) il suffit de montrer, sous les hypothèses de 2.4, que $f$ est un morphisme ouvert et même, quitte à restreindre $X$, que $f(X)$ est ouvert dans $Y$. Comme $f(X)$ est constructible, il suffit de voir que pour tout fermé irréductible $Z$ de $Y$, l'ensemble $f(X) \cap Z$ est vide ou dense dans $Z$. On peut même supposer, par changement de base, que $Z=Y$ et notre assertion résulte alors de 2.1.1(iii).

\section{Preuve du théorème $\mathbf{2 . 5}$}

Dans tout ce qui suit, $K$ désigne un corps topologique algébriquement clos.

\subsection{Le cas fini et le cas quasi-fini}

Commençons par établir 2.5 lorsque le morphisme $f$ est fini, avec une hypothèse un peu plus faible sur $K$ (toujours supposé algébriquement clos):

Proposition 3.1.1. On suppose que $K$ vérifie la condition (F). Soit $f$ : $X \rightarrow Y$ un morphisme fini et universellement ouvert de $K$-schémas de type fini. Alors l'application $f_{\text {top }}$ est ouverte.

DÉmONSTRATION. La conclusion est immédiate lorsque $X$ est réunion des images d'une famille de sections de $f$; nous allons nous ramener à ce cas par changement de base.

Fixons un entier $d$ majorant le cardinal de toutes les fibres de $f_{\text {top }}$. Considérons le diagramme suivant de $Y$-schémas finis, où les produits et puissances 
sont fibrés sur $Y$ :

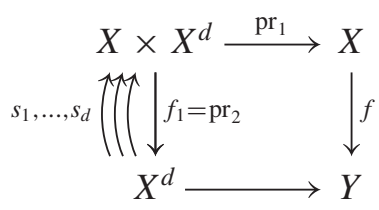

et où les sections $s_{1}, \ldots, s_{d}$ de la projection $f_{1}$ sont définies par

$$
s_{i}\left(x_{1}, \ldots, x_{d}\right)=\left(x_{i}, x_{1}, \ldots, x_{d}\right) \quad(1 \leq i \leq d) .
$$

La réunion $S$ des images des $s_{i}$ est un fermé de $X \times X^{d}$; comme $f_{1}$ est ouvert vu l'hypothèse sur $f$, il en résulte que

$$
Y^{\prime}:=\left\{t \in X^{d} \mid f_{1}^{-1}(t) \subset S\right\}
$$

est un fermé de $X^{d}$ (son complémentaire est l'image par $f_{1}$ du complémentaire de $S$ ). Vu le choix de $d, Y^{\prime}$ s'envoie surjectivement sur $Y$ : si $y \in Y(K)$, alors $f^{-1}(y)$ est de la forme $\left\{x_{1}, \ldots, x_{d}\right\}$ (avec des répétitions éventuelles) et le point $\left(x_{1}, \ldots, x_{d}\right)$ au-dessus de $y$ appartient à $Y^{\prime}$. Par restriction à $Y^{\prime}$, on obtient donc un diagramme cartésien

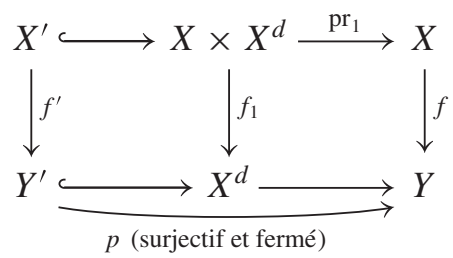

où, par construction, $X^{\prime}$ est réunion des images des sections induites par les $s_{i}$, de sorte que $f_{\text {top }}^{\prime}$ est ouverte. L'application $p_{\text {top }}$ est surjective ( $K$ est algébriquement clos) et fermée ( $K$ vérifie $(\mathrm{F})$ ) donc est un quotient topologique. Donc $f_{\text {top }}$ est ouverte en vertu de 2.3.1(ii) et du fait que le foncteur $Z \mapsto Z_{\text {top }}$ commute aux produits fibrés finis.

Par localisation étale, on en déduit le cas quasi-fini de 2.5:

Corollarie 3.1.2. Sous les hypothèses du théorème 2.5, on suppose en outre que $f$ est quasi-fini. Alors $f_{\text {top }}$ est ouverte.

Démonstration. Fixons un point $x$ de $X_{\text {top }}$, d'image $y$ dans $Y_{\text {top }}$, et montrons que $f_{\text {top }}$ est ouverte au point $x$. Si $p: Y^{\prime} \rightarrow Y$ est un morphisme étale, $y^{\prime}$ un point de $Y_{\text {top }}^{\prime}$ au-dessus de $y$, et $x^{\prime}$ le point $\left(x, y^{\prime}\right)$ de $X \times_{Y} Y^{\prime}$, il suffit de montrer l'énoncé analogue pour $x^{\prime}$ et $\mathrm{pr}_{2}: X \times_{Y} Y^{\prime} \rightarrow Y^{\prime}$, compte tenu de 2.3.1(ii) et de l'hypothèse (H) faite sur $K$. 
Or, d'après 2.3.3, il existe un tel couple $\left(Y^{\prime}, y^{\prime}\right)$ et un ouvert $Z^{\prime} \subset X \times_{Y} Y^{\prime}$ contenant le point $x^{\prime}$ et fini sur $Y^{\prime}$ : il suffit alors d'appliquer 3.1.1.

\subsection{Réduction au cas d'un morphisme quasi-fini, de but normal}

Commençons par ramener la démonstration de 2.5 au cas où $Y$ est normal. Soit $p: Y^{\prime} \rightarrow Y$ le normalisé de $Y_{\text {red }}$ et soit $f^{\prime}: X^{\prime} \rightarrow Y^{\prime}$ déduit de $f$ par le changement de base $p$ : alors $p_{\text {top }}$ est fermée et surjective donc est un quotient topologique. Ainsi il suffit, d'après 2.3.1, de démontrer que $f_{\text {top }}^{\prime}$ est ouverte.

Il nous reste donc à traiter le cas d'un morphisme $f: X \rightarrow Y$ où $Y$ est normal.

\subsection{Fin de la démonstration du théorème}

Sous les hypothèses de 2.5 , et en supposant $Y$ normal, soit $x \in X(K)$ un point quelconque: appliquant 2.3.2, on trouve un sous-schéma localement fermé irréductible $X^{\prime}$ de $X$ contenant $x$, quasi-fini et dominant sur $Y$. En outre, l'implication (ii) $\Rightarrow$ (i) de 2.3.2 montre que le morphisme induit $X^{\prime} \rightarrow Y$ est encore universellement ouvert. D'après 3.1.2, l'application induite $X_{\text {top }}^{\prime} \rightarrow$ $Y_{\text {top }}$ est ouverte, donc $f_{\text {top }}$ est ouverte au point $x$.

\section{REFERENCES}

1. Bănică, C., and Stănăşilă, O., Algebraic Methods in the Global Theory of Complex Spaces, Editura Academiei, Bucharest 1976.

2. Bănică, C., and Stănăşilă, O., Méthodes algébriques dans la théorie globale des espaces complexes 2, Troisième éd., Varia Math., Gauthier-Villars, Paris 1977.

3. Bosch, S., Half a century of rigid analytic spaces, Pure Appl. Math. Q. 5 (2009), 1435-1467.

4. Bosch, S., Güntzer,U., and Remmert, R., Non-Archimedean Analysis, Grundl. Math. Wiss. 261, Springer, Berlin 1984.

5. Bourbaki, N., Éléments de mathématique: Algèbre commutative, Masson, Paris 1985.

6. Douady, A., Flatness and privilege, Enseignement Math. (2) 14 (1968), 47-74.

7. Grothendieck, A., Éléments de géométrie algébrique. IV. Étude locale des schémas et des morphismes de schémas. II, Inst. Hautes Études Sci. Publ. Math. 24 (1965), 1-231.

8. Grothendieck, A., Éléments de géométrie algébrique. IV. Étude locale des schémas et des morphismes de schémas. III, Inst. Hautes Études Sci. Publ. Math. 18 (1966), 1-255.

9. Grothendieck, A., Éléments de géométrie algébrique. IV. Étude locale des schémas et des morphismes de schémas. IV, Inst. Hautes Études Sci. Publ. Math. 32 (1967), 1-361.

IRMAR (INSTITUT DE RECHERCHE MATHÉMATIQUE DE RENNES, UMR 6625 DU CNRS)

UNIVERSITÉ DE RENNES 1

CAMPUS DE BEAULIEU

F-35042 RENNES CEDEX

FRANCE

E-mail: laurent.moret-bailly@univ-rennes1.fr 